\title{
Rotor Position Estimation Strategy Using Artificial Neural Network for a Novel Design Transverse Flux Machine
}

\author{
Cigdem Gundogan Turker* and Feriha Erfan Kuyumcu ${ }^{\dagger}$
}

\begin{abstract}
The E-Core Transverse Flux Machine is a different design of transverse flux machines combined with reluctance principle. Determination of the rotor position is important for the movement of the ETFM by switching the phase currents in synchronism with the inductance regions of the stator windings. It is the first time that rotor position estimation based on Artificial Neural Network (ANN) is purposed to eliminate the position sensor for the ETFM. Simulation and experimental tests are demonstrated for the feasibility of the proposed estimation algorithm for the exercise bike application of the ETFM.
\end{abstract}

Keywords: Artificial neural network, Rotor position estimation, Transverse flux machine.

\section{Introduction}

The electrical machines can be categorized with flux distribution as radial, axial and transverse flux machines. In Radial Flux (RF) principle of classical electrical machines such as synchronous machines, induction machines and direct current machines, the flux lines are distributed in radial space, whereas current flows in axial direction. In case of Axial Flux (AF) Machines, flux lines cut air gap axially, whereas the current flows radially due to the windings placement. This type of electrical machines provides high torque and high power density at low speeds. Finally, the Transverse Flux (TF) principle means that the flux lines are perpendicular to the direction of the rotation. Comparing with AF and RF machines, TF machines allow an increase of the pole number to raise the torque without changing the available size of the machine. TF machines are flexible and attractive to develop in the electromagnetic design however; different topologies of TF machines have been proposed in the literature [1-7].

The E-Core Transverse Flux Machine patented by Rasmussen [8] is a different design of transverse flux machines combined with reluctance principle. The stator module of the machine is divided circumferentially with E-cores each carrying a coil wounded around centered leg. Each phase is isolated magnetically and the flux path is very short. It has important advantages such as high reliability and high torque in electrical drive systems.

The motion of the ETFM occurs with the reluctance at the air gap of the machine due to the rotor position. The rotor position must be detected to provide the current flow from one phase to the other. For reliability of the system in the industrial applications, the disadvantages of the

$\dagger$ Corresponding Author: Dept. of Electrical and Electronic Engineering, Kocaeli University, Turkey. (cdemgun@kocaeli.edu.tr)

* Dept. of Electrical and Electronic Engineering, Kocaeli University, Turkey.

Received: November 25, 2014; Accepted: April 21, 2015 mechanical sensors are eliminated with alternative sensorless methods.

The sensorless control of the electrical machines has been widely investigated in the literature similarly; different sensorless techniques have been proposed and discussed for reluctance type machines. Many of these sensorless techniques use the indirect rotor position estimation such as Flux-Current Detection Technique, Current Gradient Method, Chopping Current Detection Technique [9-11]. Furthermore, state observer, fuzzy logic and artificial neural network are implemented with the capability of operating over a wide speed range using only terminal voltage and current of the machine [12-17]. However, these techniques have been exhibited with their advantages and drawbacks depending on the typical operating principles.

Although various estimation methods and applications for the radial type reluctance machines are studied in the literature, rotor position estimation of the transverse and axial flux reluctance machines has been rarely seen. In 2007, the paper by Wisniewski is published about rotor position estimation of the axial flux permanent magnet machines with inductance based sensorless methods [18]. Also, a transverse flux linear reluctance machine using neural networks method is presented by Zongsong [19].

It is the first time that the rotor position estimation is proposed for the E-Core Transverse Flux Machine based on Artificial Neural Network (ANN) observer. The proposed system is testified for an exercise bike application, which the ETFM is placed between the pedals to give a constant load torque with the intention of providing the feeling as a real bike rider. [20] Also, the ETFM generates the electrical energy for the whole electrical equipment of the bike and stores more of that in batteries.

The paper is structured as follows: The general knowledge of the ETFM is given in the next section. Third section includes the steps of the estimation algorithm using 
ANN for rotor position estimation of the ETFM such as; determination of the training and testing data, offline verification of the ANN model and flux linkage estimation method. Simulation and experimental verification of the proposed estimation model is discussed in Section 4. Section 5 presents the waveforms of the current, voltage and results of flux estimator. In addition, the comparison of the experimental and simulation angle values is presented. Last section discusses the proposed estimation system concluding remarks.

\section{Structure of the E-Core Transverse Flux Machine}

The stator of the ETFM is based on E-shaped cores and the rotor is formed with I-shaped laminations. It has still laminated axially at the stator and rotor part without any magnets. The E-core segments have a coil around the centered leg and are assembled parallel to the rotor axis which means that it functions with the transverse flux structure. Fig. 1 shows the structure of the stator and rotor poles of the ETFM.

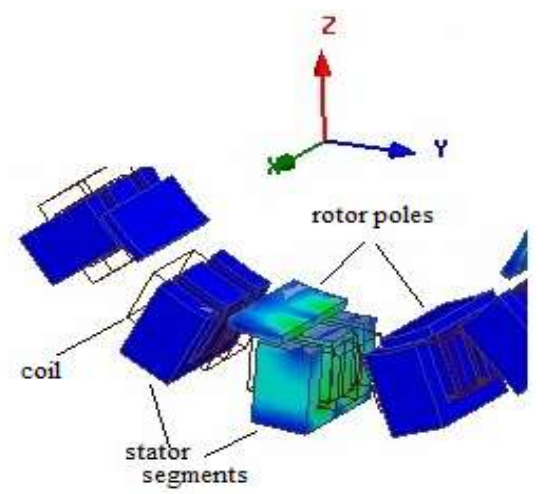

Fig. 1. Structure of the rotor and stator poles.

The movement of the ETFM is occurred in case of the reluctance generated in the air gap between stator and rotor. When the one phase of the ETFM is activated, the torque is produced with tendency of decreasing of active phase reluctance. In this way, the rotor poles are attracted to overlap with the stator poles. This position is named as aligned position of the rotor and the stator poles which there aren't produced any torque so that the other phase of the ETFM must be switched on for continuously rotation the machine rotor. The sense of rotor position is very important to have an operation and proper control over the ETFM. So, the power converter is used to switch the phases according to the rotor position.

The positive torque is produced during the rising inductance region. Conversely, the negative torque is occurred, when the phase is switched on during the decreasing inductance region, which is from aligned position to unaligned position of the rotor with a stator pole. In this way, ETFM operates in generating mode [21].

The flux linkages change according to the phase reluctance in consequence of the rotor position. Reluctance can be formulated as in (1);

$$
R=\frac{F}{\varphi}=\frac{H l}{B S}=\frac{l}{\mu S}, \mu=\mu_{0} \mu_{r}
$$

F; electromagnetic force of the stator winding, $\Phi$; magnetic flux, H; magnetic field intensity of the air gap, 1; length of the magnetic path, B; total magnetic flux density, S; area of the magnetic path, $\mu$; magnetic permeability.

The self-inductance is related with the reluctance expression given as in (2);

$$
L=\frac{\lambda}{i}=\frac{N \varphi}{i}=\frac{N^{2}}{R}
$$

$\mathrm{N}$; number of the phase winding, ; the current of the phase winding, ; magnetic flux of the machine.

Assuming of that the saturation is neglected and inductance changes linearly with rotor position, the instantaneous torque $(\mathrm{T})$ produced in the ETFM is given by;

$$
T_{e}(i, \theta)=\frac{1}{2} i^{2} \frac{d L(\theta)}{d \theta}=\frac{1}{2} i^{2} \frac{d}{d \theta}\left[\frac{N^{2}}{R}\right]
$$

\section{Rotor Position Estimation System Based on ANN}

ANN is implemented to construct a map between current, flux linkage and rotor position which are highly nonlinear characteristic of the ETFM. Required training and testing set for ANN model is comprised of magnetic flux characteristic obtained from experimental measurement. The appropriate ANN model is achieved off-line and is embedded to dSpace control processor for verifying the estimation model in progress.

Phase flux is calculated online through measurement of the phase current and phase voltage of the real system continuously. The calculated flux linkage and measured phase current are fed into ANN model to estimate the rotor position in the sampling period. As a result, the estimation process includes some steps such as; determination of the training and testing data for ANN model, off-line ANN verification, flux linkage estimation and integrating all steps within the observer model. Firstly, the system is verified with a simulation program under different performance conditions. Then, experimental setup is carried out.

Fig. 2 shows the view of the ETFM experimental system. For the exercise bike, the ETFM drive system is comprised by ETFM converter and DSP controller board, batteries 


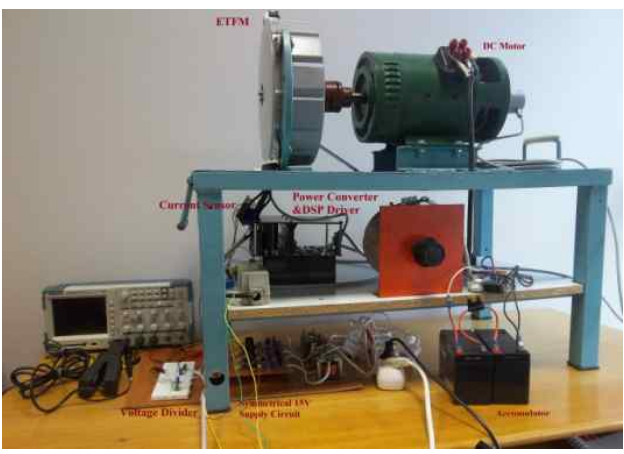

Fig. 2. Structure of the rotor and stator poles

supplying the electrical energy for the whole electrical equipments and for starting of the ETFM operation, also the DC motor as a rider that provides the different operation speeds. At the laboratory conditions, an interface program connected by PC enables different gear and level selection opportunity so that the different load conditions can be performed for simulation and experimental.

\subsection{Determination of the training and testing data}

There are several methods to obtain the magnetic characteristics of the similar electrical machines. If the geometry is well-known, finite element analysis can be used. Another way is direct or indirect measurement techniques. Flux linkage characteristics of the ETFM are highly nonlinear functions of the rotor position and current. So, it will be very difficult to set ETFM model due to its special structure [22].

Measurement method for obtaining the flux linkage characteristics is proceeded by an experimental test system. The distance of the rotor position according to a particular stator pole axis is defined as rotor position angle $(\theta)$. The electrical period of the $15 / 10$ stator/rotor pole ETFM is 360 $(3600 / \mathrm{Nr}, \mathrm{Nr}=10)$ Therefore, the rotor position angle is assumed completely unaligned position at 00 and the fully aligned position at 180 respectively.

Firstly, the resistance of the phase winding is measured without taking into consideration of temperature for the measurement procedure of flux linkage curves. The rotor position steps are moved with one degree distant and locked at the desired position. The tested phase winding is supplied with a required voltage for each current value between $0-32 \mathrm{~A}$.

The flux linkage is calculated numerically with the measured terminal winding voltage and the current based on the equation as below;

$$
\lambda(t)=\int_{t_{0}}^{t}[V(t)-R i(t)] d t+\lambda(t-1)
$$

where $\mathrm{V}$ is the winding terminal voltage, $\mathrm{I}$ is the current flowing the phase winding, is the phase flux linkage, $\mathrm{R}$ is the resistance of the phase winding. The measurement and calculation are repeated for each rotor position at the desired current values. In the Fig. 3, the flux characteristics are given for different rotor positions according to current values.

Offline Verification of the ANN Estimation Model: Training of an ANN means the determination of the connection weights of each network elements. Initially, the weight values are assigned randomly. Number of the layers and neurons for the neural network model is determined by trial and error method. In the backpropagation ANN model, inputs are attached to the network and each layer is calculated using the 'tansig' activation function until the exit layer. The weight values are updated accordingly due to the total error. Under the expected error criteria, training of the model is stopped and the last updated weight values are recorded for testing process with forward calculation method. Throughout the testing process, new input data is introduced to the network for forward calculation method.

The sufficient large training and testing data is arranged as input and output vectors from the flux linkage

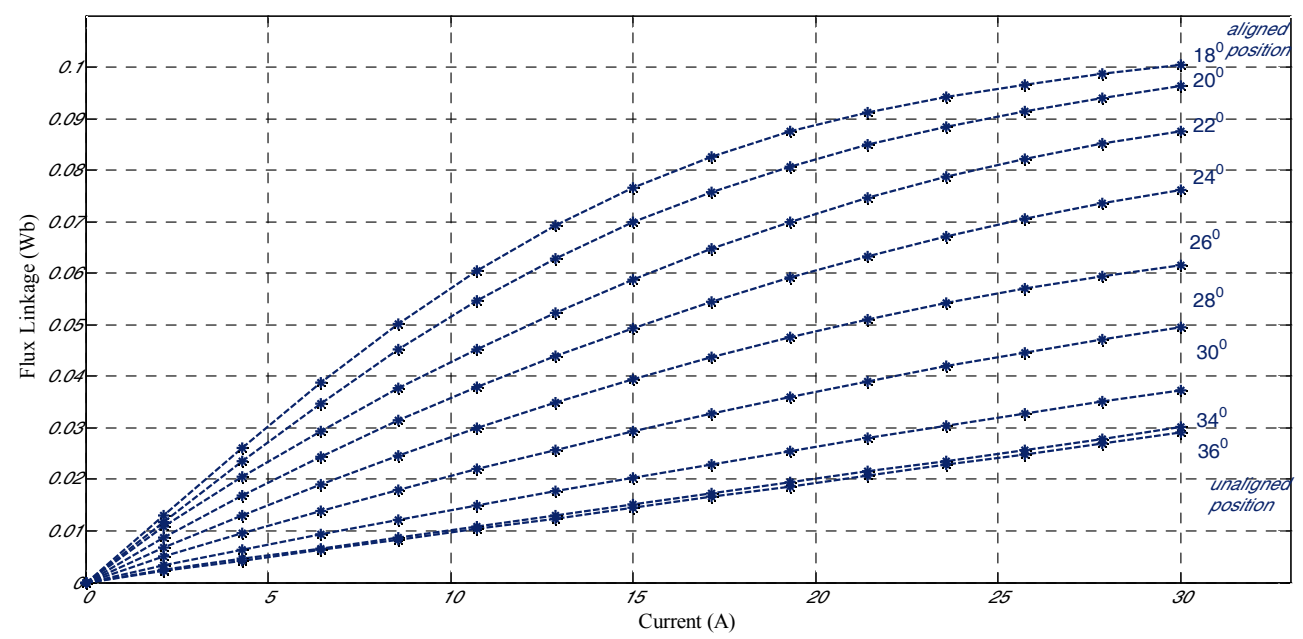

Fig. 3. The Flux linkage characteristic of the ETFM 
characteristics of the ETFM which is obtained by experimental measurement.

ANN black box is organized with the main phase winding current and its flux linkage as inputs and then rotor position as output. Matlab ANN tool is used to

Table 1. The caption must be followed by the table

\begin{tabular}{l|c}
\hline \multicolumn{1}{c}{ Properties of the ANN model } & 2 \\
\hline Number of Inputs & 1 \\
\hline Number of Outputs & 3 \\
\hline Number of Hidden Layers & 10 \\
\hline Neuron number of the first layer & 10 \\
\hline Neuron number of the second layer & 8 \\
\hline Neuron number of the third layer & Tansig \\
\hline Activation function & 0 \\
\hline Learning algorithm & 1000 \\
\hline Training target error & 0,000453 \\
\hline Epoch & \\
\hline Average error &
\end{tabular}

arrange estimation model offline. Table 1 gives the network properties.

Consequently, the scatter plot shows the comparison of the estimated rotor position values with targets in Fig. 4.

In order to evaluate the quality of the conformity to target data, the following error analysis are convenient as in (5);

$$
E_{k}=\frac{1}{N} \sum_{i=1}^{N} \frac{\left|x_{i}^{k}-\hat{x}_{i}^{k}\right|}{x_{i}^{k}}
$$

where are the target and predicted angle values, respectively of the ith data sample of each angle value, $\mathrm{k}=1,2, \ldots, \mathrm{K}$, depending on the current value of the flux characteristics, $\mathrm{i}=1,2, \ldots, \mathrm{N}$. Prediction accuracy is derived based on the mean relative error between the target and predicted values as in (6);

Table 2. Error analysis and accuracy of the ANN

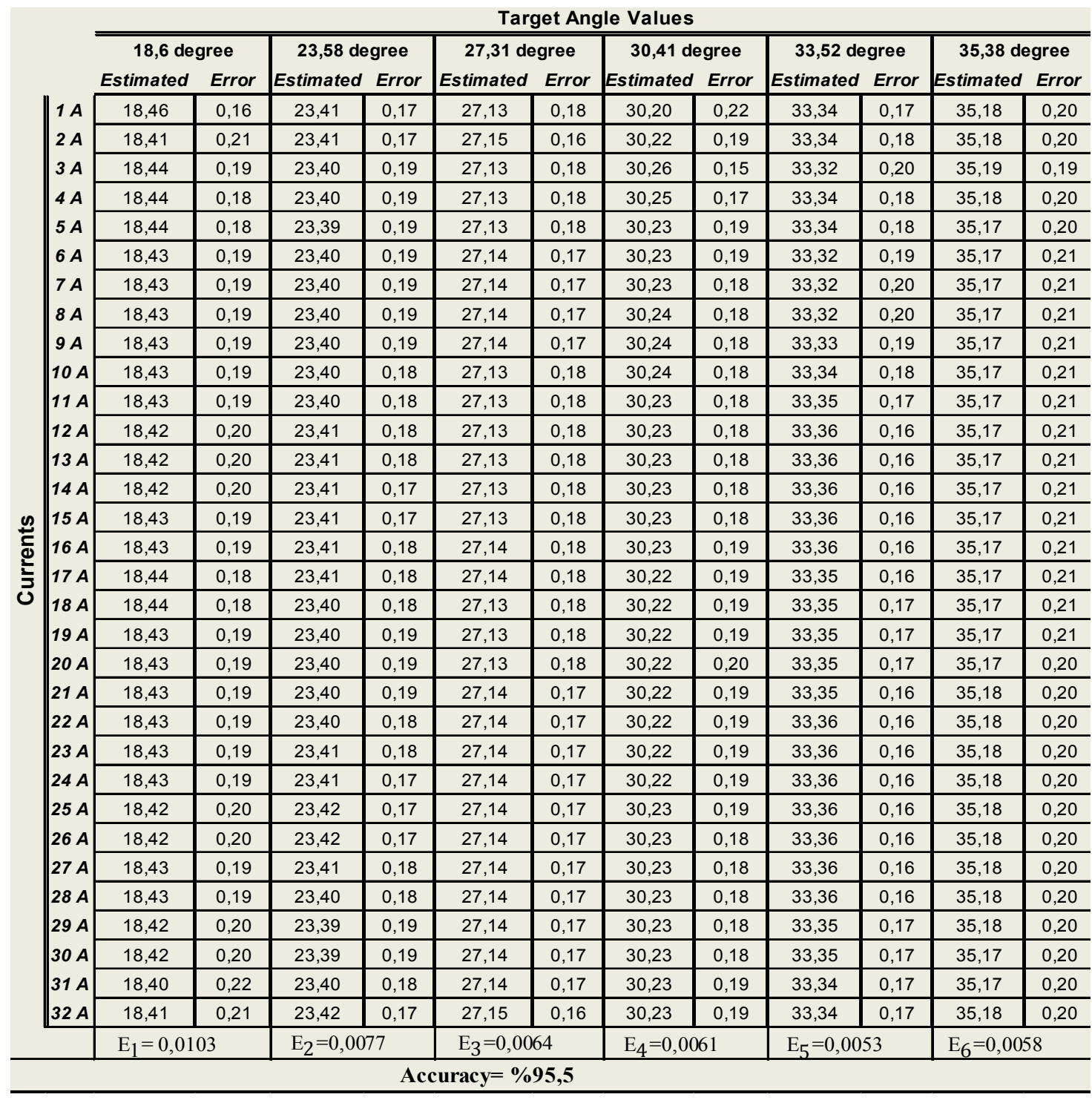




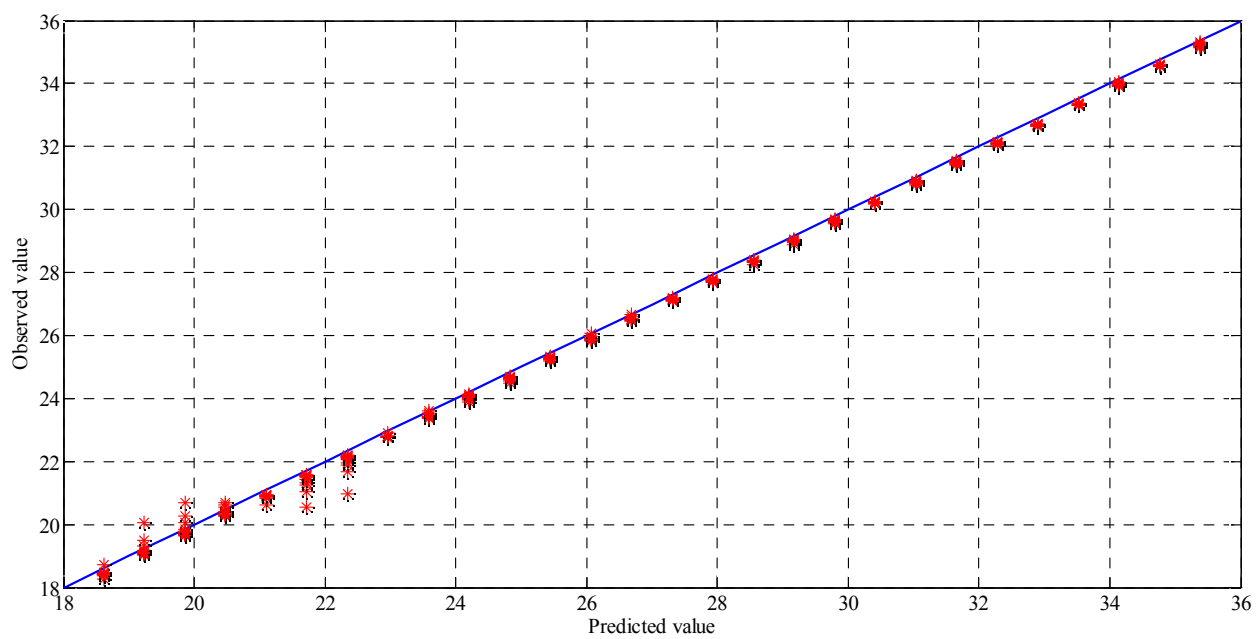

Fig. 4. Scatter Plot for the rotor position estimation ANN model

$$
\text { Accuracy }=1-\frac{1}{k} \sum_{j=1}^{k} E_{j}
$$

Table 2 shows the error analysis and accuracy of the ANN model for the rotor position estimation for $\mathrm{N}=32$ sampled data at each current value between [1-32A] and $\mathrm{K}=7$ different angle values.

\subsection{Flux linkage estimation}

Flux linkage value is used for position estimation through ANN and determination of commutation angle. It is calculated through the measurement of the phase currents and voltages in each electrical cycle of ETFM with Eq. (4).

When it is implemented in real time through a Digital Signal Processor (DSP), it has to be written in discrete time domain following as in (7);

$$
\lambda(t)=[V(t)-i(t) R] T+\lambda(t-1)
$$

When the current of the active phase goes down to zero, the flux linkage will be zero. Therefore, it numerically limits the error due to rounding, digitization and its accumulation. In order to implement real time integration of the flux linkage estimator, the initial value of flux linkage is reset to zero periodically.

\section{Simulation and Experimental Verification of the Proposed Estimation System}

It is demonstrated the accuracy and effectiveness of the proposed position estimator with the simulation and the experimental implementation of the ETFM system. Simulation of the estimation model for rotor position is performed under Matlab/Simulink and then experimental system is set up. The reference simulation model is executed with the same parameters and sampling frequency of the input variables as in the real time operation of the experimental system. Simulation includes the reference torque table that is applied to the rider according to the gear and level value selection, also the reference current and phase turn-on angle tables in accordance with the system working.

The phase voltage and the current of the appropriate phase are measured and the corresponding flux linkage is calculated using Euler integrator form given as Eq. (7) at each sampling time of the system in progress. Afterward, the flux linkage and the current values from the energized phase are fed to the ANN rotor position estimation model. An optical incremental encoder is installed on the machine shaft to provide the rotor position information just for using comparison and verification. Fig. 5 shows the outline of the developed measurement system for the proposed method.

The estimation method based on ANN is executed with the "DS-1104" controller board that is plugged into a slot of PC. It is featured with four simultaneous 16-bit and four 12-bit ADC channels, eight 12-bit DAC channels, 2 encoder output, $250 \mathrm{MHz}$ operating frequency. An I/O board provides an interface between the DS1104 board and the measurement sensors. The data processing is performed inside the Dspace microprocessor as block diagram given

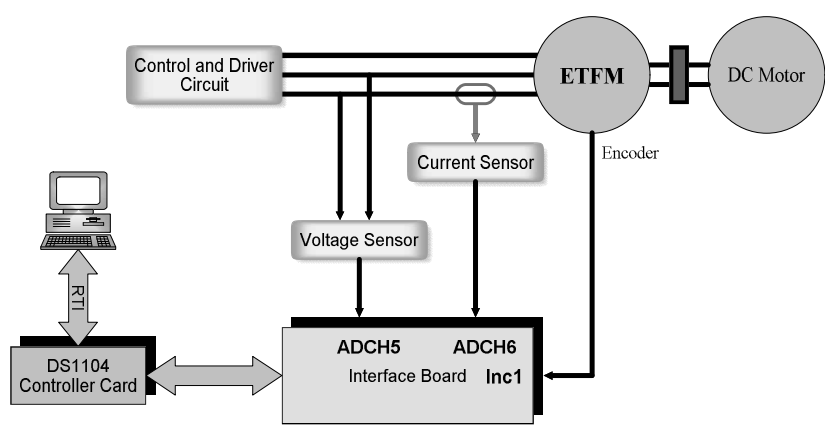

Fig. 5. Schematic diagram of the estimation system 


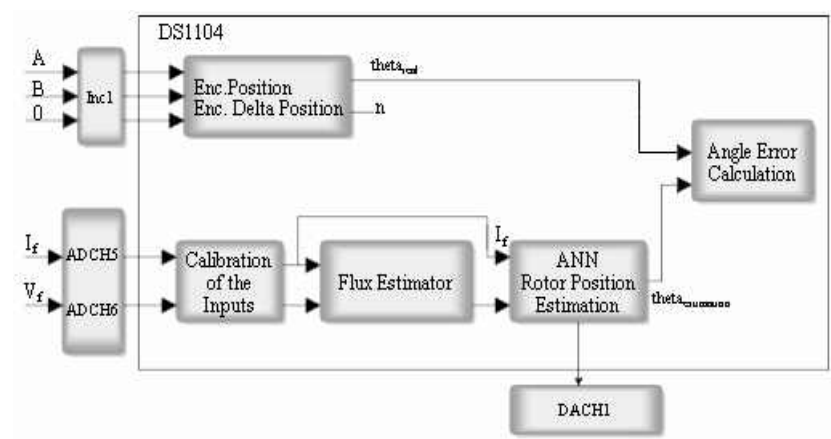

Fig. 6. Block diagram of the real time data processing inside the Dspace in the Fig. 6.

DS1104 is appropriate for motion control. It uses real time interface implementation software to generate and download the real-time code to the board. The model of the plant and control algorithm is designed simply by drawing its block diagram using graphical Real Time Interface (RTI) Matlab/Simulink. The real time code is generated directly from Simulink models using the Real Time Workshop toolbox. The data and the system parameters in the real time platform can be observed and modified using Control Desk program which enables to create graphic user interfaces using simple GUI elements like push-buttons, displays, labels, etc. Controller parameters can be adjusted

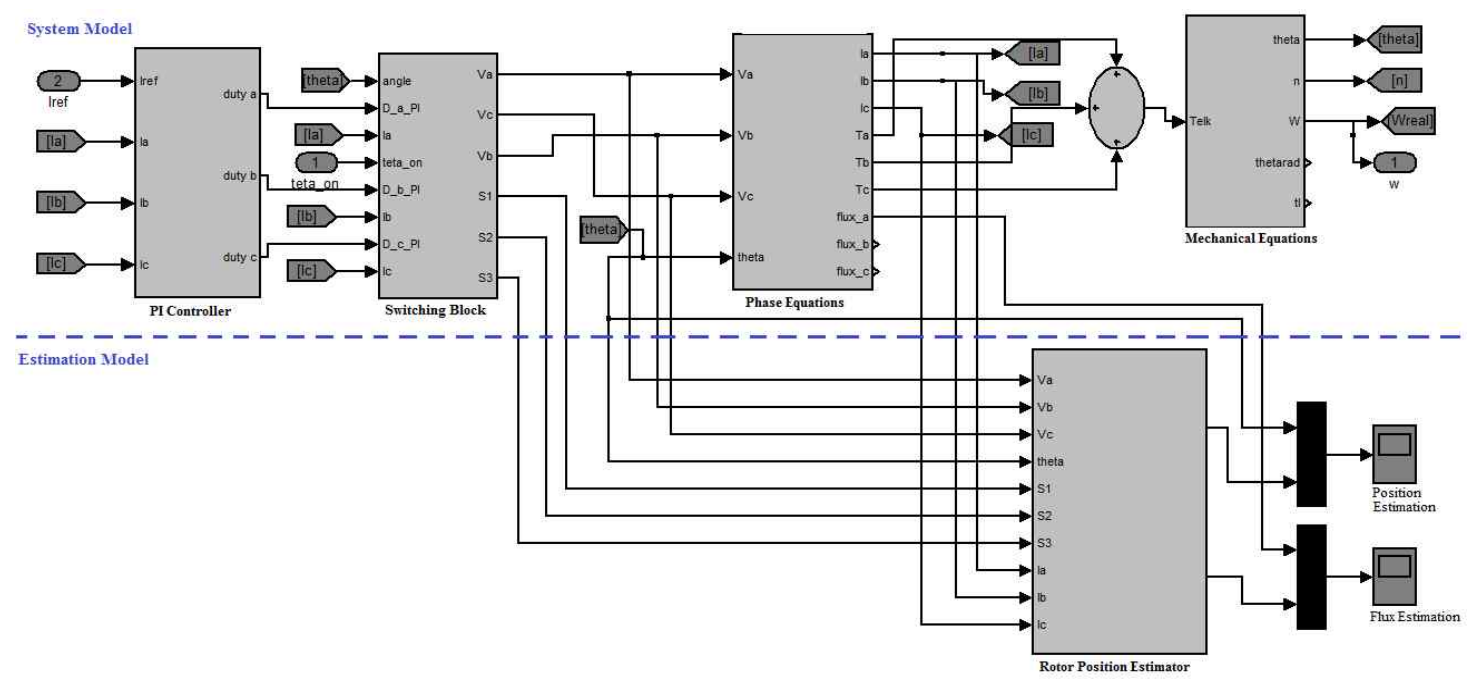

Fig. 7. The simulation model of the ETFM sensorless rotor position estimation system

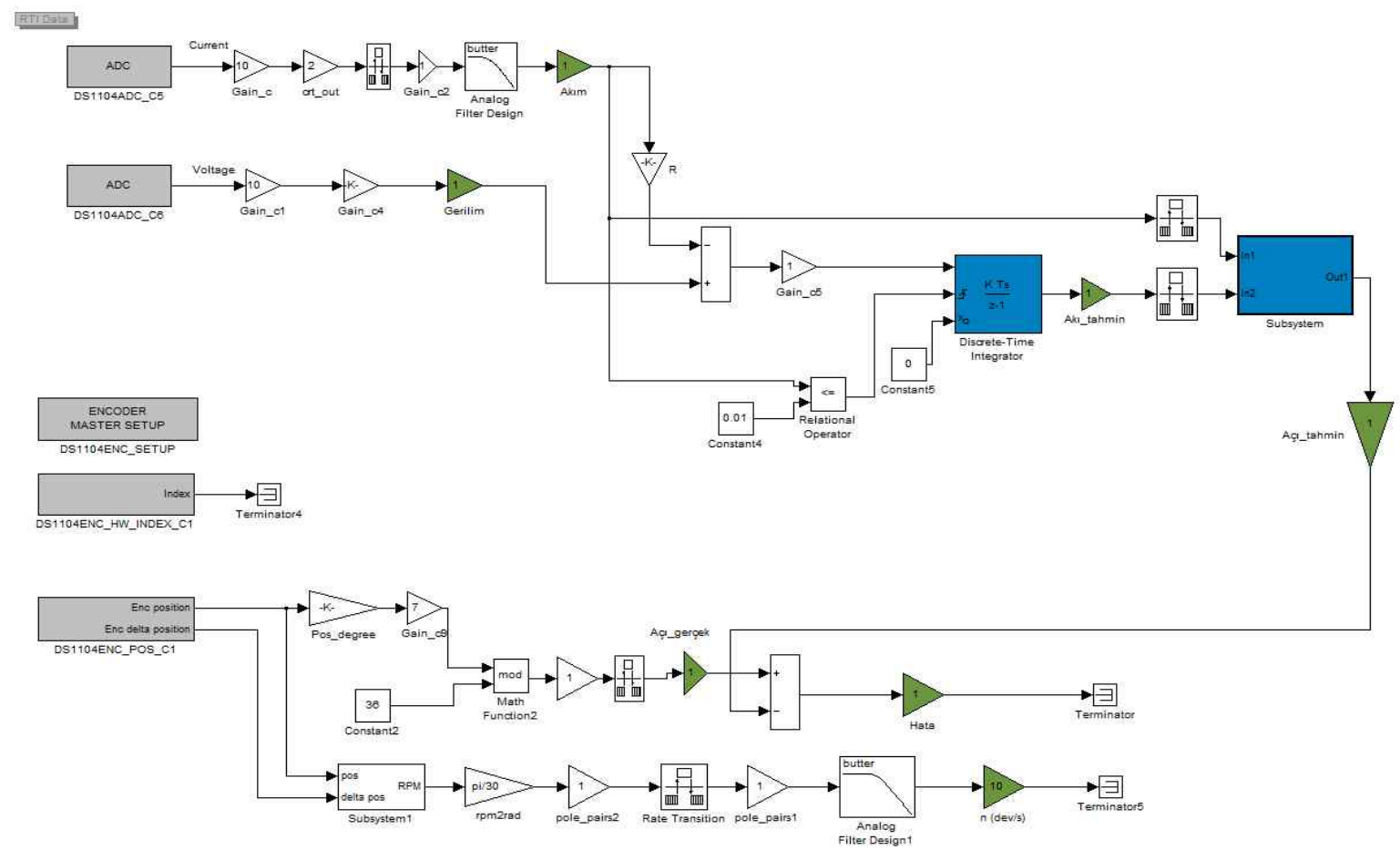

Fig. 8. The graphic interface of the control algorithm using RTI 


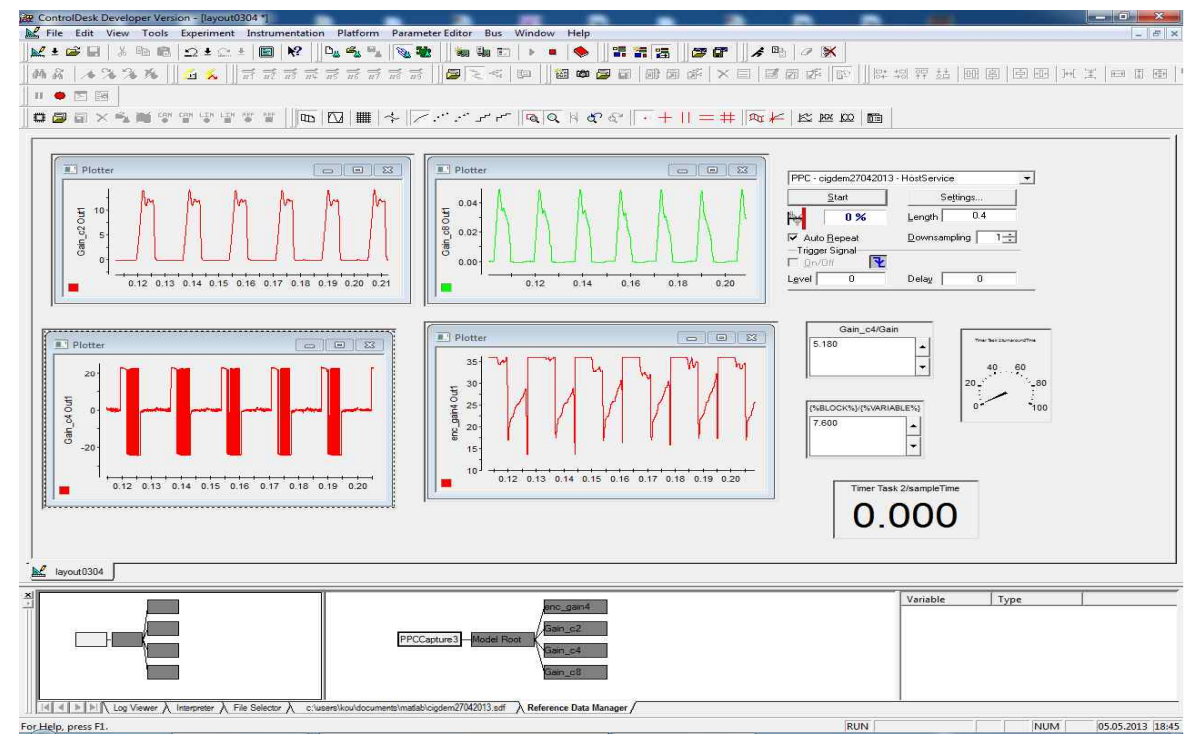

Fig. 9. The real time platform showing the current, voltage, flux linkage waveforms and predicted angle values

online without having to rebuild and download a new Simulink model to the board.

The whole system blocks of the ETFM and the position estimator including the ANN system and flux estimation algorithm are shown in Fig. 7. The graphic interface of the control algorithm using RTI of Simulink is showed in Fig. 8. The real time platform observing the current, voltage, flux linkage waveforms and predicted angle values at Control Desk Program is given in Fig. 9.

\section{Results of The Proposed Estımation System}

For sensing the angular position of the ETFM, an incremental position encoder is mounted with the drive system and connected to the digital encoder input of the DS1104 interface board. In this way, the comparison between actual position measured by the sensor and estimated by the proposed method is attained under different load conditions of the exercise bike application.

A most appropriate phase should be selected to continue the estimation. ANN was trained for the best estimation interval within [180-360] that ETFM works as generator. Two adjacent phases of the ETFM have 12-degree phase shift. This suggests that each phase can hand over its estimation duty to the next phase at $\theta=180+120=300$. It must be combined with the estimated position of three individual phases into one electrical period.

In Fig. 10 (a), the current and voltage waveforms are given for gear $=4$, level $=7, \omega=375 \mathrm{~d} / \mathrm{dk}$. The flux estimation for experimental and simulation is compared in fig. 10.b. In the fig. 10.c, it is shown the comparison of the rotor position angle for one excited phase.

In Fig. 11(a), the current and voltage waveforms are given for gear $=5$, level $=8, \omega=290 \mathrm{rpm}$. The flux estimation for experimental and simulation is compared in Fig. 11(b).

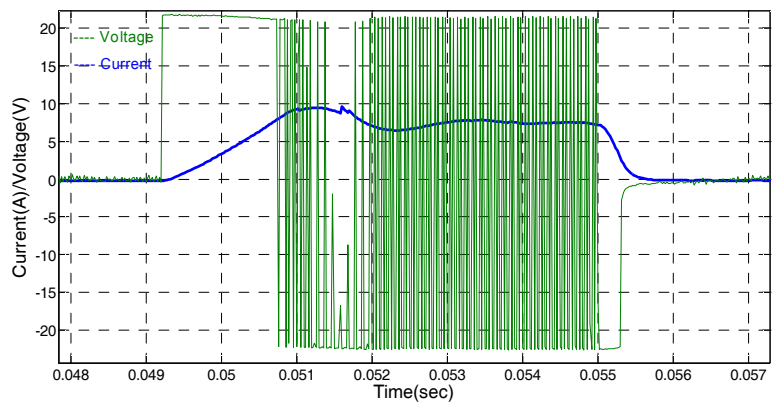

(a)

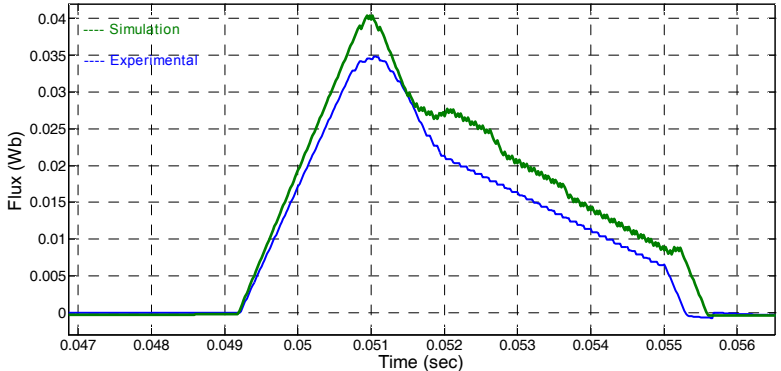

(b)

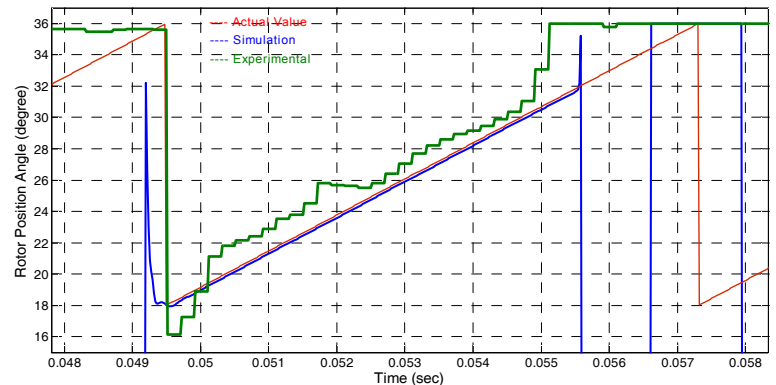

(c)

Fig. 10. Gear=4, Level=7, $\omega=375 \mathrm{rpm}$ : (a) The current and voltage waveform; (b) Comparison of the flux estimation; (c) The comparison of the rotor position angle. 


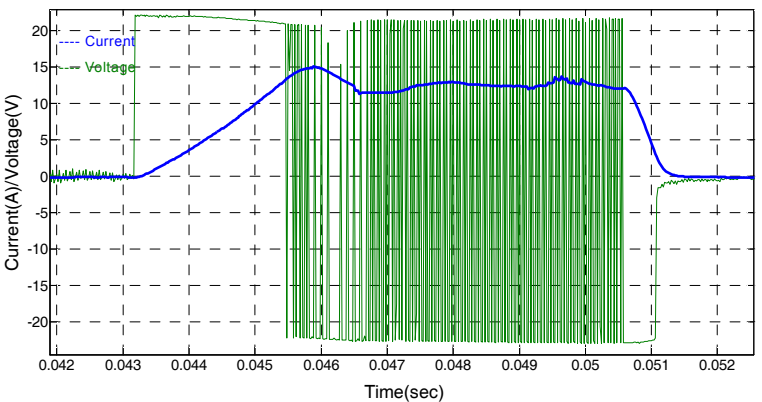

(a)

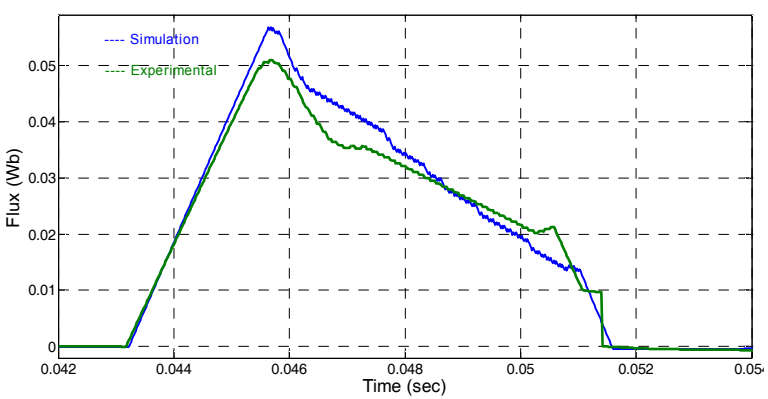

(b)

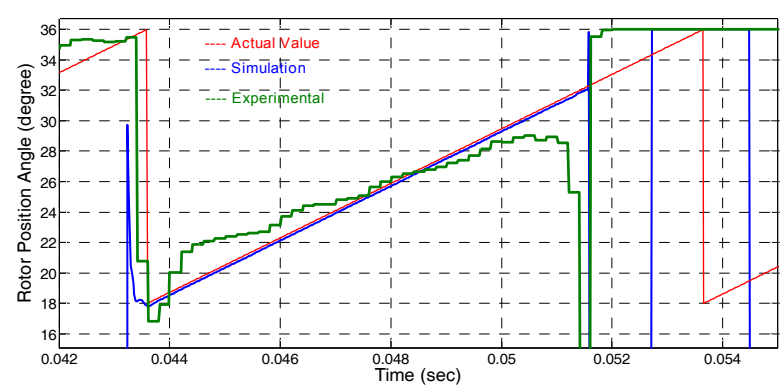

(c)

Fig. 11. Gear $=5$, Level $=8, \omega=290 \mathrm{rpm}$ : (a) The current and voltage waveforms of the experimental system; (b) Comparison of the flux estimation; (c) The comparison of the rotor position angle.

In the Fig. 11(c), it is shown the comparison of the rotor position angle for one excited phase.

\section{Conclusion}

The paper proposed to estimate the rotor position of the ETFM by a sensorless method based on ANN. Simulation and experimental tests are demonstrated the feasibility of using this technique to eliminate the encoder of the system. The proposed method is succeeded easily with the dSpace controller card DS1104 as a result of the application with Matlab Real Time Interface Program of the proposed control algorithm. The sensorless rotor position algorithm based on ANN is verified with the real time angle value obtained from the encoder mounted with drive system.

Firstly, the flux linkage is indirectly obtained by integration from the inputs, which are the voltage and current signals and fed into the ANN position estimator as input. The flux linkage estimator is important for rotor position estimation but the errors in the voltage and current measurements due to noise, sensor offset, sensor nonlinearity, quantization or integration errors such as truncation errors, can result significant inaccuracies in the flux linkage. Also, the accuracy of the expert system largely depends upon the accuracy of the training data measured experimentally especially for neural network model.

Sampling frequency is adjusted as $50 \mathrm{kHz}$ to capture the voltage and current waves. Although the higher frequency can be given better results, executed ANN algorithm and flux integrator process limits the adjustment probability of the frequency particularly for the DS1104 card.

Actually, the simulation and experimental results shows that the proposed ANN system can be utilized to estimate the rotor position angle of the ETFM if the errors derived from noise, quantization, sampling frequency can be reduced.

\section{Appendix A}

Parameters of the ETFM

Ns (Number of stator phase)

$\mathrm{Nr}$ (Number of rotor pole)

Phase number

$0.09 \mathrm{ohm}$

$\mathrm{R}$ (One phase resistance)

$32 \mathrm{~A}$

In (nominal current)

$660 \mathrm{rpm}$

wn (Nominal speed)

Lmin (minimum inductance)

$0,9543 \mathrm{mH}$

\section{Acknowledgements}

Authors gratefully thank to Anders Hedeager Pedersen from E-Power Technology and to the Aalborg University for their support for the application system.

\section{References}

[1] H. Weh, "Transverse flux machines in drive and generator applications", IEEE International Symposium on Electric Power Engineering, Stockholm, Sweden, 1998.

[2] G. Henneberger, M. Bork, "Development of a new transverse flux motor", IEE Collegium on New Topologies for Permanent Magnet Machines, London, England, 18 June 1997

[3] R. Kruse, G. Pfaff, C. Pfeiffer, "Transverse flux reluctance motor for direct servo drive applications", IEEE Industry Applications Conference, St. Louis, USA, 12-15 October 1998.

[4] Y. H. Jeong, D. H. Kang, J. M. Kim, et al., "Design of transverse flux motor with permanent magnet 
shield", IEEE International Symposium on Industrial Electronics, Busan, South Korea, 12-16 June 2001.

[5] W.M.Arshad, P. Thelin, T. Backstrom, et al., "Use of transverse-flux machines in a free-piston generator", IEEE Trans. on Ind. Applications, 40 (4), pp. 1092$1100,2004$.

[6] Z. Rahman, "Evaluating radial, axial and transverse flux topologies for in wheel motors", Power electronics in Transportation, Novi, USA, Oct. 2004.

[7] C. Junghwan, D. Kang, J. Lee, et al., "Development of transverse flux linear motor with permanentmagnet excitation for direct drive applications", IEEE Trans. on Magnetics, 41 (5), pp.1936-1939, 2005.

[8] P. O. Rasmussen, "Transverse flux machine with Stator Made of E-shaped laminations", United States patent US7312549, 2007.

[9] P. P. Acarnley, R. J. Hill, C. W. Hooper, "Detection of rotor position in stepping and switched reluctance motors by monitoring of current waveforms", IEEE Trans. Ind. Electron, IE 32, pp. 215-222, 1985.

[10] M. Ehsani, I. Husain, "Elimination of discrete position sensors and current in switched reluctance motor drives", IEEE Trans. on Industry Applications, 28, pp. 128-135, 1992.

[11] S. R. Macmin, P. M. Szensny, W. J. Rzeson, et al., "Application of sensor integration techniques to switched reluctance motor drives", IEEE Transactions on Industry Applications, 28 (6), pp. 1339-1344, 1992.

[12] A. Lumsdaine, J. H. Lang, M. J. Balas, "A state observer for variable reluctance motors: analysis and experiments", Nineteenth Asilomar Conference on Circuits, Systems and Computers, Pacific Grove, USA, 6-8 November 1985.

[13] C. Elmas, H. Selaya-De-La Parra, "Application of a full order extended Luenberger observer for a position sensorless operation of a switched reluctance motor drive", IEEE Proc. Control Theory and Applications, 143, (5), pp. 401-408, 1996.

[14] Y. J. Zhan, C. C. Chan, K. T. Chau, "A Novel sliding mode observer for indirect position sensing of switched reluctance motor drives", IEEE Transactions on Industrials Electronics, 46 (2), pp. 390-397, 1999.

[15] N. S. Islam, I. Husain, R. J. Veilette, "Design and performance analysis of sliding mode observers for sensorless operation of switched reluctance motors", IEEE Trans. Cont. Syst. Technology, 11, pp. 383-389, 2003.

[16] A. D. Cheok, Z. Wong, "Fuzzy logic rotor position estimation based switched reluctance motor DSP drive with accuracy enhancement", IEEE Transactions Power Electron, 20, pp. 908-921, 2005.

[17] E.Meşe, D. A. Torrey, “An Approach for sensorless position estimation for switched reluctance motors using artificial neural networks", IEEE Trans. Power Electron, 17, pp. 66-75, 2002.

[18] J. Wisnewski, "Pole position identification of per- manent magnet axial flux motor using PIPCRRM sensorless method", European Conf. On Power Electronics and Applications, Aalborg, Denmark, 2-5 September 2007.

[19] C. Zhongsong, G. Baoming, T. Aribal, "Neural network based position sensorless control for transverse flux linear SRM", Advances in Neural Network, Lecture Notes in Computer Science, 4493, pp. 73-79, 2007.

[20] P. O. Rasmussen, G. Runolfsson, T. A. Thorsdottir, et al., "E-core transverse flux machine with integrated fault detection system", International Conference on Electrical Machines and Systems, Beijing, China, 2011.

[21] I. Kioskerides, C. Mademlis, "Optimal Efficiency Control of Switched Reluctance Generators", IEEE Transactions on Power Electronics, 21(4), 2006.

[22] C. Gundogan Turker, F. Erfan Kuyumcu, "The Modelling of The E-Core Transverse Flux Machine Based on Neural Network", International Review of Electrical Engineering, 5 (4), pp.1477-1487, 2010.

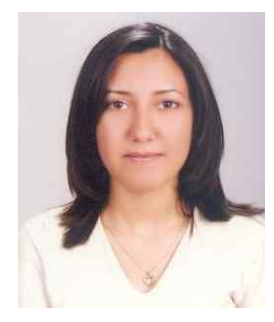

Cigdem Gundogan Turker She received the M.S. and Ph.D. degrees in electrical engineering from Kocaeli University, Turkey, in 2004 and 2013, respectively. Her interests are electrical modelling and analysis of the electrical machines, power systems, drives and control.

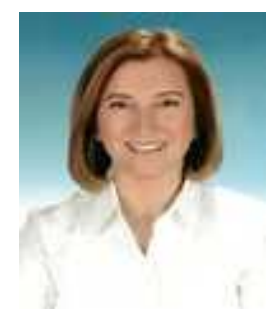

Feriha Erfan Kuyumcu She received the B.S. degree from Istanbul Technical University, Istanbul, Turkey, in 1984 and the M.S. and Ph.D. degrees from Yildiz Technical University, Istanbul, Turkey, in 1987 and 1992, respectively, all in electrical engineering. She joined Kocaeli University in 1993 and is currently a Professor at the Department of Electrical Engineering. Her current research interests include electric propulsion systems, electric machines and drives and power systems modeling and simulation. Her principal research concerns the design and control of switched reluctance motor. 